\title{
Numerical Simulations of Turbulent Dynamos
}

\author{
Axel Brandenburg \\ NORDITA, Blegdamsvej 17, DK-2100 Copenhagen $\emptyset$, Denmark; and \\ Department of Mathematics, University of Newcastle upon Tyne, NE1 \\ $7 R U, U K$
}

\begin{abstract}
Using a periodic box calculation it is shown that, owing to helicity conservation, a large scale field can only develop on a resistive timescale. This behaviour can be reproduced by a mean-field dynamo with $\alpha$ and $\eta_{t}$ quenchings that are equally strong and 'catastrophic'.
\end{abstract}

\section{Introduction}

After the original papers by Vainshtein \& Cattaneo (1992) and Cattaneo \& Vainshtein (1991) about 'catastrophic' $\alpha$ and $\eta_{\mathrm{t}}$ quenching respectively, there has been a continuous debate about the existence and relevance of $\alpha$-effect dynamos in astrophysics, i.e. in the high $R_{\mathrm{m}}$ regime. Their results were supported by calculations that did not however have dynamo action (see also Cattaneo \& Hughes 1996). In the present paper we show, using a periodic box calculation that, owing to helicity conservation, a large scale field can only develop on a resistive timescale, and that this behaviour can be reproduced by a mean-field dynamo with $\alpha$ and $\eta_{\mathrm{t}}$ quenchings that are equally strong and 'catastrophic' in the sense that quenching sets in once the large scale field reaches the fraction $R_{\mathrm{m}}^{-1 / 2}$ of the equipartition value.

\section{Helicity constraint and catastrophic quenching}

Large scale magnetic field generation has long been associated with helicity (e.g., Moffatt 1978, Krause \& Rädler 1980). However, the large scale fields generated by turbulence with kinetic helicity generally possess also magnetic helicity. This is a conserved quantity and characterizes the linkage of magnetic flux structures with themselves. If the magnetic helicity is zero initially within a certain volume, it can only change if there is a loss of magnetic helicity of preferentially one sign through the boundaries (Blackman \& Field 2000, Kleeorin et al. 2000). Magnetic helicity can also change resistively, allowing the large scale magnetic helicity to grow while the small scale magnetic helicity is being dissipated, but this is a slow process if the magnetic Reynolds number is large. Using only helicity conservation and the assumptions that the large scale field, $\bar{B}$, possesses helicity, one can show that (Brandenburg 2000, hereafter B2000)

$$
\left\langle\overline{\boldsymbol{B}}^{2}\right\rangle \approx k_{\mathrm{f}} \ell_{A B} B_{\mathrm{eq}}^{2}\left[1-\exp \left(-2 \eta k_{1}^{2}\left(t-t_{\text {sat }}\right)\right] .\right.
$$


Here, $t_{\mathrm{sat}}=\lambda^{-1} \ln \left(B_{\mathrm{fin}} / B_{\mathrm{ini}}\right)$ is the time when the field at small and intermediate scales saturates, $B_{\text {ini }}$ and $B_{\text {fin }}$ are initial and final field strengths, $\lambda$ is the kinematic growth rate of the dynamo, $B_{\text {eq }}$ is the equipartition field strength, and $k_{\mathrm{f}}$ is the wavenumber of the forcing.

Equation (1) is rather general and independent of the actual model of field amplification. If the field is not fully helical, then $\ell_{A B}=|\langle\bar{A} \cdot \overline{\boldsymbol{B}}\rangle| /\left\langle\overline{\boldsymbol{B}}^{2}\right\rangle$ will be less than $1 / k_{1}$, where $k_{1}=2 \pi / L$ is the smallest possible wavenumber. Thus, the final field amplitude will be reduced. In any case however, the time it takes to reach this final amplitude scales with the resistive time, $\left(\eta k_{1}^{2}\right)^{-1}$, where $\eta$ is the microscopic (not the turbulent!) value.

Equation (1) suggests that, after $t=t_{\text {sat }}$ the large scale field grows at first linearly, $\left\langle\bar{B}^{2}\right\rangle=2 \eta k_{1}^{2}\left(t-t_{\text {sat }}\right)$, and then slowly approaches $B_{\text {fin }}$. In Fig. 1 we compare the evolution of $\left\langle\overline{\boldsymbol{B}}^{2}\right\rangle$ for Run 3 of B2000 with the result of Eq. (1). In this run we have $B_{\text {ini }}=2 \times 10^{-9}, B_{\mathrm{fin}}=0.342$, and $\lambda=0.067$, so $t_{\text {sat }}=283$.

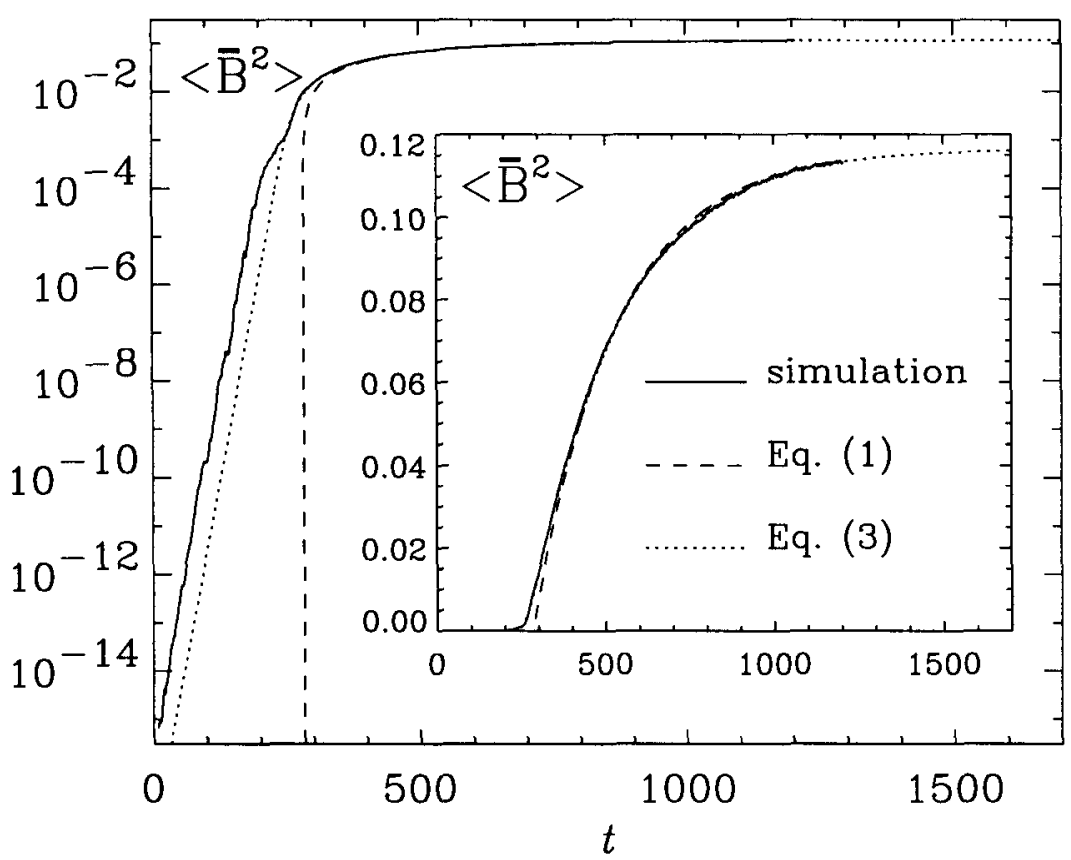

Figure 1. Evolution of $\left\langle\overline{\boldsymbol{B}}^{2}\right\rangle$ for Run 3 (solid line) compared with Eq. (1) (dashed line). The dotted line gives the result for an $\alpha^{2}$ meanfield model (discussed below). The inset shows the evolution on a linear scale. Note the excellent agreement between simulation and the analytic fits during the saturation phase. $u_{\mathrm{rms}}=0.18, k_{1}=1, k_{\mathrm{f}}=5$, $\eta=0.002$. 


\section{How to model this?}

Exactly the same resistive asymptotic behaviour can be reproduced using an $\alpha^{2}$-dynamo with simultaneous $\alpha$ and $\eta_{\mathrm{t}}$-quenching of the form

$$
\alpha=\frac{\alpha_{0}}{1+\alpha_{B} \bar{B}^{2} / B_{\mathrm{eq}}^{2}}, \quad \eta_{\mathrm{t}}=\frac{\eta_{\mathrm{t} 0}}{1+\eta_{B} \overline{\boldsymbol{B}}^{2} / B_{\mathrm{eq}}^{2}},
$$

where $\alpha_{B}=\eta_{B}$ is assumed. Assuming that the magnetic energy density of the mean field, $\overline{\boldsymbol{B}}^{2}$, is approximately uniform (which is well obeyed in the simulations) we can obtain the solution $\overline{\boldsymbol{B}}=\overline{\boldsymbol{B}}(t)$ of the $\alpha^{2}$-dynamo equations in the form (B2000)

$$
\bar{B}^{2} /\left(1-\bar{B}^{2} / B_{\text {fin }}^{2}\right)^{1+\lambda / \eta k_{1}^{2}}=B_{\text {ini }}^{2} e^{2 \lambda t},
$$

where $\lambda=\left|\alpha_{0}\right| k_{1}-\eta_{\mathrm{T} 0} k_{1}^{2}$ is the kinematic growth rate, and $\eta_{\mathrm{T} 0}=\eta+\eta_{\mathrm{t} 0}$. The reason the field saturates in spite of simultaneous quenching of $\alpha$ and $\eta_{\mathrm{t}}$ is that there remains still the microscopic diffusivity $\eta$, which is not quenched, preventing therefore indefinite growth. This determines the saturation field strength in terms of the quenching parameters $\alpha_{B}$ and $\eta_{B}$ and yields

$$
\alpha_{B}=\eta_{B}=\frac{\lambda}{\eta k_{1}^{2}}\left(\frac{B_{\mathrm{eq}}}{B_{\mathrm{fin}}}\right)^{2},
$$

where $B_{\mathrm{eq}}^{2}=\mu_{0}\left\langle\rho u^{2}\right\rangle$ is characteristic of the magnetic energy at small scales. To a good approximation the spectral energy in the small scales is close to that in the large scales, so $k_{1} B_{\mathrm{fin}}^{2} \approx k_{\mathrm{f}} B_{\mathrm{eq}}^{2}$. Thus, $\alpha_{B} \approx \lambda /\left(\eta k_{1} k_{\mathrm{f}}\right)$. Since $\lambda$ scales with $u_{\text {rms }} k_{\mathrm{f}}$ we have $\alpha_{B} \approx u_{\mathrm{rms}} /\left(\eta k_{1}\right)$, so the quenching coefficient scales with the magnetic Reynolds number based on the box scale, not the forcing scale as one might have expected. Taking into account factors of order unity, e.g. $\lambda \approx 0.07 u_{\mathrm{rms}} k_{\mathrm{f}}$ (where $k_{\mathrm{f}}=5$ ) and $(2 \pi)^{-1}$, we have $\alpha_{B}=\eta_{B} \approx R_{\mathrm{m}} / 60$, where $R_{\mathrm{m}}=u_{\mathrm{rms}} L / \eta$.

We conclude that Eq. (3) provides an excellent fit to the numerical simulations (Fig. 1) and one might therefore be tempted to extrapolate to astrophysical conditions. However, real astrophysical systems have open boundary conditions and we now need to know whether this could alleviate the issue of very long timescales for the mean magnetic field.

\section{References}

Blackman, E. G., \& Field, G. F. 2000, ApJ 534, 984

Brandenburg, A. 2000, ApJ, astro-ph/0006186 (B2000)

Cattaneo, F., \& Hughes, D. W. 1996, Phys. Rev. E 54, R4532

Cattaneo, F., \& Vainshtein, S. I. 1991, ApJ 376, L21

Kleeorin, N. I, Moss, D., Rogachevskii, I., \& Sokoloff, D. 2000, A\&A 361, L5

Krause, F., \& Rädler, K.-H. 1980, Mean-Field Magnetohydrodynamics and Dynamo Theory (Pergamon Press, Oxford)

Moffatt, H. K. 1978, Magnetic Field Generation in Electrically Conducting Fluids (CUP, Cambridge)

Vainshtein, S. I., \& Cattaneo, F. 1992, ApJ 393, 165 